\title{
Correction: Gaglio et al. Performances of Different Metabolic Lactobacillus Groups during the Fermentation of Pizza Doughs Processed from Semolina. Fermentation 2018, 4, 61
}

\author{
Raimondo Gaglio ${ }^{1}$, Antonio Alfonzo ${ }^{1, *}$, Noemi Polizzotto ${ }^{1}$, Onofrio Corona ${ }^{1}{ }^{\circledR}$, Nicola Francesca ${ }^{1}{ }^{1}$, \\ Giuseppe Russo $^{2}$ (D), Giancarlo Moschetti ${ }^{1}$ (D) and Luca Settanni ${ }^{1}$ (D) \\ 1 Dipartimento Scienze Agrarie e Forestali, Università degli Studi di Palermo, Viale delle Scienze 4, \\ 90128 Palermo, Italy; raimondo.gaglio@unipa.it (R.G.); noemipoli@hotmail.it (N.P.); \\ onofrio.corona@unipa.it (O.C.); nicola.francesca@unipa.it (N.F.); giancarlo.moschetti@unipa.it (G.M.); \\ luca.settanni@unipa.it (L.S.) \\ 2 Consorzio di Ricerca “Gian Pietro Ballatore”, Z.I. Dittaino s.n.c., 90040 Assoro (Enna), Italy; \\ giusepperusso@ilgranoduro.it \\ * Correspondence: antonio.alfonzo@unipa.it
}

check for updates

Citation: Gaglio, R.; Alfonzo, A.; Polizzotto, N.; Corona, O.; Francesca, N.; Russo, G.; Moschetti, G.; Settanni,

L. Correction: Gaglio et al.

Performances of Different Metabolic Lactobacillus Groups during the Fermentation of Pizza Doughs Processed from Semolina.

Fermentation 2018, 4, 61. Fermentation 2021, 7, 143. https://doi.org/ $10.3390 /$ fermentation7030143

Received: 14 July 2021

Accepted: 22 July 2021

Published: 4 August 2021

Publisher's Note: MDPI stays neutral with regard to jurisdictional claims in published maps and institutional affiliations.

Copyright: (c) 2021 by the authors. Licensee MDPI, Basel, Switzerland. This article is an open access article distributed under the terms and conditions of the Creative Commons Attribution (CC BY) license (https:// creativecommons.org/licenses/by/ $4.0 /)$.
The authors wish to make the following corrections to this paper [1]:

In the abstract section, there are some errors. We mistakenly indicated that Lactobacillus sanfranciscensis, Lactobacillu brevis, and Lactobacillus rossiae are part of the facultative homofermentative group and Lactobacillus plantarum, Lactobacillus graminis, and Lactobacillus curvatus are part of the obligate heterofermentative group (lines 3-4 in the abstract), but the opposite is true. Lactobacillus plantarum, Lactobacillus graminis, Lactobacillus curvatus are facultative, and Lactobacillus sanfranciscensis, Lactobacillu brevis, and Lactobacillus rossiae are obligate.

The correct version is as follows:

The main hypothesis of this work is that facultative and obligate heterofermentative Lactobacillus species can differently impact the final characteristics of pizza. The objective was to evaluate separately the behaviors of the obligate heterofermentative species (OHS), such as Lactobacillus sanfranciscensis, Lactobacillus brevis, and Lactobacillus rossiae, and the facultative heterofermentative species (FHS), including Lactobacillus plantarum, and Lactobacillus curvatus, in the sourdoughs to be used for pizza production. The production of the experimental pizzas was carried out with semolina (Triticum turgidum L. ssp. durum). The acidification process - which was followed by $\mathrm{pH}$, total titratable acidity (TTA), and lactic acid bacteria (LAB) development-indicated for all of the experimental trials that the kinetics is comparable to those of the controls. The fermentation quotient of the FHS trial was particularly higher than that of the other trials, including the control production performed with a sourdough inoculum used in an artisanal bakery. The dominance of the added strains indicated the clear persistence of L. sanfranciscensis PON100336, L. brevis 200571, and L. plantarum PON100148 in the obligate-facultative heterofermentative species (OFHS) trial. The pizzas were baked without seasoning in order to investigate weight loss, color, morphology, and a generation of volatile organic compounds (VOCs). The data showed the differences among trials regarding the inocula. Eight classes of VOCs were detected in the pizza samples with aldehydes, esters, alcohols, and acids as major compounds. The sensory attributes were significantly different for the judges and the pizzas. The multivariate statistical approach found marked differences among the trials. The results indicated that the application of mixed cultures of the facultative heterofermentative species of Lactobacillus determined high quality pizzas.

The authors would like to apologize for any inconvenience caused to the readers by these changes. 
Author Contributions: Conceptualization, A.A. and L.S.; Methodology, A.A., G.R. and R.G.; Software, N.F.; Validation, N.F. and G.M.; Formal Analysis, A.A., O.C. and N.P.; Investigation, A.A. and R.G.; Data Curation, A.A., O.C. and R.G.; Writing-Original Draft Preparation, L.S.; Writing-Review \& Editing, A.A. and L.S.; Supervision, G.M. and L.S.; Project Administration, L.S. All authors have read and agreed to the published version of the manuscript.

Funding: This work was financially supported by the Project for Industrial Research and Training, PON01_02249, “Application of molecular biotechnologies and pro-technological microorganisms for the characterisation and valorisation of dairy and bakery chains of typical products", of the Italian Ministry of Education, University, and Research (CUP: B11C11000430005).

Institutional Review Board Statement: Not applicable.

Informed Consent Statement: Not applicable.

Data Availability Statement: All data included in this study are available upon request by contacting the corresponding author.

Conflicts of Interest: The authors declare no conflict of interest.

\section{Reference}

1. Gaglio, R.; Alfonzo, A.; Polizzotto, N.; Corona, O.; Francesca, N.; Russo, G.; Moschetti, G.; Settanni, L. Performances of Different Metabolic Lactobacillus Groups During the Fermentation of Pizza Doughs Processed from Semolina. Fermentation 2018,4 , 61. [CrossRef] 